\title{
On the luminosity function of planetary nebulae
}

\author{
D. Schönberner, R. Jacob and M. Steffen \\ Astrophysikalisches Institut Potsdam, 14482 Potsdam, Germany \\ email: deschoenberner@aip.de, msteffen@aip.de
}

\begin{abstract}
A new assessment of the physics of the luminosity function of planetary nebulae is presented, based on our grid of nebular evolutionary sequences computed with a 1D radiationhydrodynamics code. The nebular evolution is followed from the formation stage in the vicinity of the asymptotic-giant branch across the Hertzsprung-Russell diagram until the white-dwarf domain is reached, for various central-star models coupled to different initial envelope configurations. Along each sequence the relevant line luminosities of the nebulae are computed and analyzed. Our models predict that reasonably dense initial circumstellar envelopes with central stars of slightly above $0.6 \mathrm{M} \odot$ will remain nearly optically thick and are able to provide the observed $5007 \AA$ cutoff luminosity. We cannot support the claim of Marigo et al. (2004) according to which only planetary nebulae with central stars of $>0.7 \mathrm{M}_{\odot}$ are able to provide sufficient $5007 \AA$ line emission to account for the bright end of the luminosity function.
\end{abstract}

Keywords. hydrodynamics - radiative transfer - planetary nebulae: general - stars: AGB and post-AGB

\section{Introduction}

Despite its use for nearly 20 years, the physical basis of the planetary nebula luminosity function (PNLF) is still mysterious and subject to controversal interpretations. The main uncertainty is related to the question whether planetary nebulae $(\mathrm{PNe})$ are optically thick or thin for Lyman continuum photons because the efficiency of converting stellar UV photons into optical line emission is heavily dependent on the corresponding optical depth. A PN is a complex system even in spherical approximation. Any model has to account for (i) a rapidly evolving central star, with time scales comparable to the dissipation time scale of the nebula, (ii) a shock wave pattern set up by the stellar radiation field and the stellar wind. Our approach aiming at understanding the basic physics of the luminosity function is based on radiation-hydrodynamics simulations with the proper initial and boundary conditions, with all the physical processes treated fully time-dependently.

\section{Modelling and results}

For the study of the luminosity function we selected appropriate model simulations from our extensive study on PN evolution (Perinotto et al. 2004). These simulations are based on our 1D radiation-hydrodynamics code which treats ionization, recombination, heating and cooling fully time-dependently. The cooling function is composed of the individual contributions of all the ions considered ( $\mathrm{H}, \mathrm{He}, \mathrm{C}, \mathrm{N}, \mathrm{O}, \mathrm{Ne}, \mathrm{Cl}, \mathrm{S}, \mathrm{Ar})$. Further details are given in Perinotto et al. (1998) and Marten \& Szczerba (1997).

The evolutionary behaviour of our sequences in terms of $M(5007)$ is shown in Fig. 1, and the corresponding individual luminosity functions are illustrated in Fig. 2. 

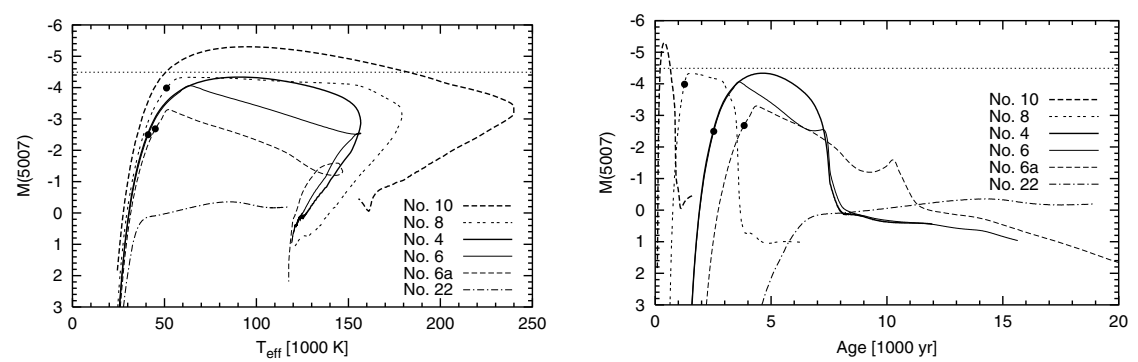

Figure 1. Predicted evolution of $M(5007)$ vs. stellar effective temperature (left) and post-AGB age $($ right $)$ for sequences with different central-star masses: $0.565 \mathrm{M}_{\odot}$ (No. 22), $0.595 \mathrm{M}_{\odot}$ (No. 6a), 0.605 $\odot$ (No. $4 \& 6), 0.625 \mathrm{M}_{\odot}$ (No. 8), and $0.696 \mathrm{M}_{\odot}$ (No. 10). The shape of the curves reflects the evolution of the central stars across the HR diagram: the upturn corresponds to the horizontal evolution, the downturn to the final luminosity fading. The filled dots indicate the moments when the nebular models become optically thin in the Lyman continuum. Note that the sequences No. 4 \& 10 remain optically thick during their whole computed evolution, and that the optically thick part of sequence No. 6 coincides with sequence No. 4 . The models of sequence No. 22 reach the optically thin stage very early. The local brightness spikes seen in the sequences with optically thin nebular shells are due to recombination. The observed bright $M(5007)$ cut-off is also indicated (dotted).
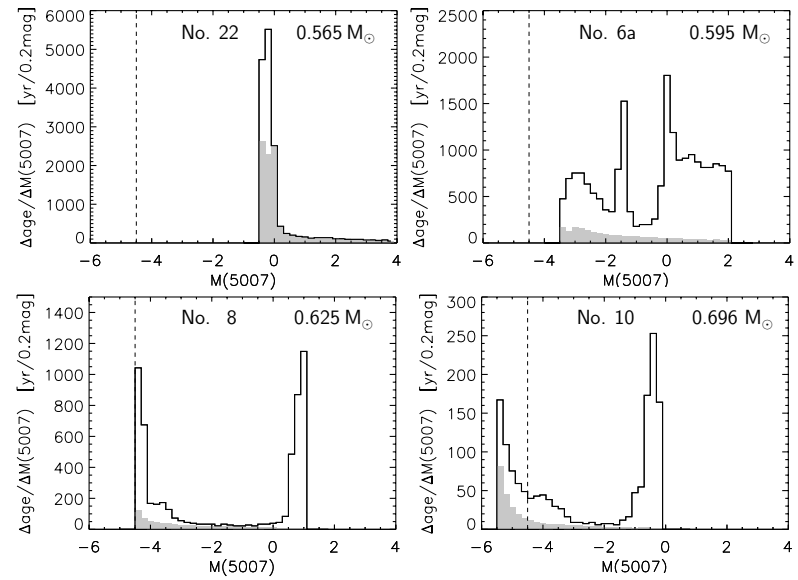

Figure 2. Total times spent per absolute magnitude bin of 0.20 mag for selected sequences with different central stars. The evolution proceeds from faint magnitudes towards a maximum brightness and then back again. The gray areas represent the contributions from the evolution towards the bright cutoff at $M(5007)=-4.5$ (vertical dashed line). There appears to be a faint cut-off at $M(5007) \simeq 2$ caused by re-ionization and slowing down of the stellar fading.

In conclusion we find that (i) the maximum $5007 \AA$ emission occurs either at $T_{\text {eff }} \simeq$ $90000 \mathrm{~K}$, or earlier when the PN becomes optically thin (see Fig. 1), and that (ii) PNe with central stars of $\simeq 0.62 \mathrm{M}_{\odot}$ are able to reach $M(5007) \approx-4.5$. Both statements, based entirely on our hydrodynamical models, are in conflict with the results of Marigo et al. (2004) which, however, are drawn from much simpler nebular models.

\section{References}

Marigo, P., Girardi, L., Weiss, A., Groenewegen, M.A.T. \& Chiosi, C. 2004, A $\& A$ 423, 995

Marten, H. \& Szczerba, R. 1997, A\&A 248, 590

Perinotto, M., Kifonidis, K., Schönberner, D. \& Marten, H. 1998, A\&A 332, 1044

Perinotto, M., Schönberner, D., Steffen, M. \& Calonaci, C. 2004, A\& A 414, 993 